Harv Rev Psychiatry. 2015 ; 23(2): 112-121. doi:10.1097/HRP.0000000000000070.

\title{
Alcohol Use Disorders in Pregnancy
}

\author{
Jeffrey DeVido, MD ${ }^{1,2}$, Olivera Bogunovic, MD², and Roger D. Weiss, MD² \\ ${ }^{1}$ University of California, San Francisco, Department of Psychiatry, San Francisco, CA \\ ${ }^{2}$ McLean Hospital, Division of Alcohol and Drug Abuse, Harvard Medical School, Belmont, MA
}

\section{Abstract}

Alcohol use disorders (AUD) during pregnancy are less prevalent than in non-pregnant women, but they can create a host of clinical challenges when encountered. Unfortunately, there is little research information available to guide clinical decision-making in this population. Drinking alcohol during pregnancy can have negative consequences on both fetus and mother, but there is controversy regarding the volume of alcohol consumption that correlates with these consequences. There is little evidence to support the use of pharmacologic interventions for AUD during pregnancy. Similarly, there are few data to guide management of alcohol detoxification in pregnant women, and the use of benzodiazepines (the mainstay of most alcohol detoxification protocols) in pregnant women is controversial. Despite a lack of robust data to guide management of AUDs in pregnancy, clinicians must nonetheless make management decisions when confronted with these challenging situations. Therefore, this paper reviews the epidemiology of AUDs in pregnancy, and the pharmacologic management of both AUDs and alcohol withdrawal in pregnant women, to better inform clinicians about what is known about managing these co-occurring conditions.

\section{Introduction}

Data from respondents in the 2012 National Survey on Drug Use and Health (NSDUH) revealed that $8.5 \%$ of pregnant women in America consumed at least one alcoholic drink in the previous 30 days, $2.7 \%$ drank five or more drinks during one episode in the previous 30 days (defined as binge drinking), and $0.3 \%$ had five or more drinks on the same occasion five or more times in the last 30 days (defined as heavy drinking). ${ }^{1}$ The survey further found that the rate of alcohol consumption in pregnant women has been decreasing $(11.6 \%$ in 2006-2007 versus $8.5 \%$ in 2012), ${ }^{2}$ despite increasing rates of alcohol consumption in women, overall (31.7\% versus $33.2 \%$ in 2002 and 2012, respectively). ${ }^{3}$ Relative to their non-pregnant peers, pregnant women consume overall less alcohol (among non-pregnant women, $55.1 \%$ drank at least once in the last thirty days, $24.5 \%$ reported binge drinking, and $5.3 \%$ endorsed heavy drinking). ${ }^{4}$ However, the NSDUH does not include military or

Contact: Jeffrey DeVido MD; University of California, San Francisco, 401 Parnassus Avenue, Box 0984, LPPI, San Francisco, CA 94143-0984, phone: 415.476.7716, fax: 415.476.7371, jeffrey.devido@ucsf.edu.

This manuscript has not previously been presented at any meetings, nor has it been previously published. This manuscript represents original material. All authors have read and approved this version of the manuscript. 
homeless populations of women; therefore, it is conceivable that the prevalence of alcohol consumption in American pregnant women may be higher than the NSDUH suggests.

These data suggest that pregnancy may be a time of increased motivation to decrease or stop drinking, and relatively higher rates of success in doing so. In support of this assertion, a 2006 Norwegian population-based study demonstrated that of nearly 1,500 women surveyed, $85 \%$ altered their alcohol consumption upon learning of their pregnancy, with fetal well-being cited as the primary reason for the change. ${ }^{5}$ Furthermore, the United States' Substance Abuse and Mental Health Services Administration (SAMHSA) cites decreasing alcohol consumption as pregnancy progresses, with 2013 rates of consumption in the first, second, and third trimesters falling from $17.9 \%$ to $4.2 \%$ to $3.7 \%$, respectively. ${ }^{6}$

Despite data suggesting that alcohol consumption during pregnancy is comparably lower than non-pregnant peers, that is little consolation when confronted with a pregnant woman who presents with an AUD. These patients present a host of management challenges such as: how to best advise women of the risks to them and their fetus posed by alcohol consumption, screening strategies unique for pregnant women with AUDs, preferred pharmacologic and behavioral interventions, risks posed by alcohol withdrawal, and best approaches for management of alcohol withdrawal in pregnancy.

\section{Methods}

\section{Search Strategy}

For this review, PubMed was used to search for relevant English language articles, using keywords specific to the multiple dimensions of this review. No time limit was specified on the search up until April 29,2014. However, articles not available in electronic format were excluded owing to resource restrictions. No further exclusions were applied. For risks posed by alcohol consumption during pregnancy, the following keywords were used: alcohol AND pregnancy AND risks, alcohol AND birth AND defects, alcohol AND pregnancy AND consequences, alcohol AND pregnancy AND health policy. For screening for alcohol use disorders in pregnancy, keywords were: alcohol AND pregnancy AND screening tools, alcohol disorders AND screening tools. Keywords for the section on pharmacologic interventions for AUDs in pregnancy were as follows: pregnancy AND naltrexone, pregnancy AND disulfiram, pregnancy AND acamprosate. Behavioral interventions for AUDs in pregnant women keywords were: alcohol AND pregnancy AND behavioral interventions, alcohol AND pregnancy AND psychotherapy. Alcohol withdrawal in pregnancy section keywords were: alcohol AND pregnancy AND withdrawal, alcohol AND pregnancy AND withdrawal risks. Finally, management of alcohol withdrawal in pregnancy keywords were: alcohol AND pregnancy AND withdrawal treatment, pregnancy AND benzodiazepines. Results obtained in each search were scanned for relevance, and pertinent manuscripts were individually reviewed for additional relevant citations potentially missed through the initial search parameters. 


\section{What Are The Risks Posed by Alcohol Consumption During Pregnancy?}

In 1967, the French pediatrician Paul Lemoine first characterized alcohol as a teratogen. Lemoine described 127 cases of similar anomalies observed in children born of mothers with "chronic alcoholism," although the exact volume of alcohol consumption is not specified. ${ }^{7,8}$ Jones and Smith in 1973 built upon Lemoine's work by defining the specific spectrum of dysmorphologies associated with alcohol use during pregnancy and coining the term Fetal Alcohol Syndrome (FAS) to describe the pattern of "craniofacial, limb, and cardiovascular defects associated with prenatal-onset growth deficiency and developmental delay." 9

Subsequently, researchers have characterized other hazards tied to in utero alcohol exposure, even in children who do not present the full spectrum of FAS dysmorphologies, leading to the development of a broader definition of the pathology: fetal alcohol spectrum disorders (FASD). For example, low birth weight, preterm birth, and small for gestational age, ${ }^{10}$ spontaneous abortions, ${ }^{11}$ behavioral problems, ${ }^{12}$ developmental delay, ${ }^{13}$ and cognitive deficits ${ }^{14}$ have all been linked in a dose-response pattern to alcohol use during pregnancy. However, despite these linkages between increased alcohol consumption during pregnancy and physiologic, behavioral, and cognitive deficits, there is no consensus on at what specific volume of alcohol consumption these risks increase, since many researchers examining this relationship have found little or no consistent correlation between "low" or "moderate" amounts of alcohol and these pathologies, ${ }^{15,16,17}$ while others have. ${ }^{18}$

Much of the review literature examining the relationship between volume of maternal alcohol consumption and fetal/infant risks has been qualitative in nature; ${ }^{19}$ however, a few meta-analyses on the topic have been undertaken. ${ }^{17,20,21}$ Meta-analysis offers the advantage of pooled data across studies and "permits the computation of effect size estimates across multiple studies and formal testing of hypothesized moderator variables" (Testa, et al, 2003, page 295). ${ }^{17}$

In their meta-analysis of the literature, Testa, et al (2003) examined infant mental development as assessed through the widely-used and standardized Mental Health Development Index (MDI) and Bayley Scales of Infant Development tools at ages 6, 12 and 18-26 months of age. Scores on these assessment scales were then correlated with daily maternal alcohol use of less than one standard drink per day, between 1 and 2 drinks per day, and greater than 2 drinks per day. The data demonstrated a complex picture, with a general trend notable for a negative linear effect on MDI with amount of alcohol consumed. However, when covariates and socioeconomic status were factored in, the trend became less clear (which may also be a reflection, in part, of the inherent limitations of the MDI to pick up differences in mental development at the different ages examined). The authors point out that it is particularly striking that no clear trend emerged, despite the large number of qualitative reviews available and their relative impact on social policy. ${ }^{17}$

In their meta-analysis of the literature, Polygenis, et al (1997), examined the relationship between "moderate" maternal alcohol consumption (defined as less than 2 standard alcoholic drinks per day) during the first trimester of pregnancy and risk of fetal 
malformations. ${ }^{20}$ The authors' analysis included data from over 20,000 exposed infants in Canada, and they found no increased risk between moderate first trimester drinking and incidence of fetal malformations. They cite a potential limitation of the study being possible recall bias. However, it can stand to reason that many post-delivery mothers may actually be more prone to under-report drinking habits due to social stigma. ${ }^{22}$ Under-reporting of amount of alcohol consumed would mean that the lack of risk association found in this analysis actually represented a lack of association with a potentially higher amount of alcohol consumed, casting further doubt on the risks of lower amounts of alcohol consumption.

In distinction to the equivocal findings in the meta-analyses of Polygenis and Testa, Sayal, et al, (2007) performed a prospective population-based study that examined the relationship between self-reports of amount and frequency of alcohol use during the first trimester and the presence of clinically significant behavioral or emotional problems at 47 and 81 months (parental report, $n=9086$ and 8046, respectively), and 93 to 108 months of age (teacher report, $\mathrm{n}=5648$ ). Behavioral and emotional problems were assessed through the standardized Strengths and Difficulties Questionnaire (SDQ). The data revealed that maternal consumption of $<1$ drink per week during the first trimester was independently associated with behavioral or emotional problems in girls detectable first at 47 months, and persisting through later time points. ${ }^{18}$ While not definitive, these data add to the uncertainty about socalled "safe" levels of drinking during pregnancy. These data suggest that perhaps even lowlevel of alcohol exposure at crucial in utero developmental points, in certain particularly vulnerable populations (in this case, female gender) may have long-term deleterious behavioral and/or emotional consequences.

From a public health standpoint, this uncertainty about the level of safe alcohol consumption (if safe at all) has been problematic, and national health services have varied in their recommendations to pregnant women and their healthcare providers regarding the risks of consuming alcohol in pregnancy. For instance, since enactment of the Anti-Drug Abuse Act of 1988, the United States Surgeon General's Office has advised the following regarding alcohol consumption in pregnancy: "According to the Surgeon General, women should not drink alcoholic beverages during pregnancy because of the risk of birth defects." 23 Many governments, such as those of Australia, The Netherlands, and Canada have recently joined the United States in advising total abstinence from alcohol as the safest option in pregnancy, while other countries, like the United Kingdom, have advised abstinence as the safest option, but offer recommendations for low-level alcohol use, if the pregnant woman should decide to drink. ${ }^{24}$ Confounding the issue are different definitions of one "standard" alcoholic drink in different countries. For example, the standard drinks (in grams of ethanol) in the United States, Australia, Canada, and the United Kingdom are 14g, 10g, 13.6g, and $8 \mathrm{~g}$, respectively. 25

Despite the relatively similar public health recommendations among several countries (Canada, Australia, and the United States), the rates of alcohol consumption in pregnant women in these countries none the less can vary greatly. For example, Canadian studies have indicated that $15 \%$ of pregnant women consume alcohol. ${ }^{26}$ Data from Australia suggest $47.3 \%$ of women drink alcohol while pregnant but prior to learning of their 
pregnancy, and $19.5 \%$ of women continue to drink after learning of their pregnancy. ${ }^{27}$ As highlighted above, United States survey data indicate that $17.9 \%$ of pregnant women consume alcohol in the first trimester. ${ }^{6}$ This suggests that there are perhaps additional complex local social or other factors weighing in on a pregnant woman's decision to drink alcohol.

One might suspect that the incidence of maternal alcohol consumption in the United Kingdom might be higher relative to the countries with more prohibitive guidelines, but the rates of first, second, and third trimester alcohol consumption in the United Kingdom have been estimated at $11.5,4.0$, and $1.8 \%$, respectively. ${ }^{28}$ These estimated rates actually appear less than those estimated in the United States (17.9\% to $4.2 \%$ to $3.7 \%$, listed earlier), although it can be difficult to compare rates directly, as the systems for measuring rates may have differed significantly from country to country.

Data suggest that the incidence of FASD might be higher in those countries with higher incidence of maternal alcohol consumption. For example, the estimated rates of FASD in the United States range from 0.2 to 1.5 cases per 1000 live births ${ }^{29}$ ), while the rate of FASD in Australia (where the rate of alcohol consumption during pregnancy is perhaps more than in the United States, as described above), stands at 6 per 1000 live births. ${ }^{30}$ This difference may be reflective of overall higher rates of alcohol consumption during pregnancy in Australia, but these data may additionally suggest more subtle risks associated with drinking patterns rather than just simply exposure to alcohol during pregnancy. For instance, as mentioned above, survey data suggest that $47 \%$ of Australian women consume alcohol while pregnant but before they knew of their pregnancy, suggesting much higher rates of alcohol consumption among Australian women than United States women (33.2\%). ${ }^{3}$ Therefore, different rates of alcohol consumption early in pregnancy may account for the significant differences in rates of FASD between the United States and Australia.

These data cast further uncertainty into understanding the risks associated with alcohol consumption in pregnancy and deleterious development effects. Moreover, this may appear to throw additional uncertainty into the utility of public health guidelines regarding alcohol consumption in pregnancy; however, the variable rates in alcohol use among countries with different public health guidelines may be more reflective of differences in social norms that are informed by a multiplicity of factors (not just government policy statements).

Nonetheless, the power of social norms in shaping decision making regarding drinking in alcohol is substantial. One Australian study, for example, highlighted that the single most important factor for women in determining alcohol consumption during pregnancy is social norms. ${ }^{31}$

In this light, governmental public health policy statements therefore could be seen as just one thread in the fabric of formation of social norms. In the presence of sometimes uncertain data regarding the risks of alcohol in pregnancy, perhaps the safest recommendation remains abstinence. To this end, it should be noted that no data suggest any beneficial effects on fetal or maternal health or development posed by alcohol use during pregnancy. Allowing abstinence-only public health recommendations to further integrate into the fabric of social norms may, in time, further decrease rates of maternal alcohol consumption. 
In summary, alcohol is a known teratogen, and its use during pregnancy can result in a host of major physical, psychological, and cognitive problems, many of which are subtle and not yet well-characterized. However, the dose at which the risks to fetal development rise is uncertain. It seems that social norms (which can be shaped by public health policy positions) can have a positive impact on women's decisions to drink or not during pregnancy. Having clear governmental guidelines on the matter may also reduce clinician confusion about how to best advise women of the risks of alcohol use during pregnancy.

\section{Screening for AUDs During Pregnancy}

Several screening tools for AUDs have been empirically validated, such as the CAGE, ${ }^{32}$ Alcohol Use Disorders Identification Test (AUDIT) and its revision, the AUDIT-C, ${ }^{33}$ the Michigan Alcoholism Screening Test (MAST) ${ }^{34}$ Tolerance, Worried, Eye-opener, Amnesia, K/Cut down attempts (TWEAK), ${ }^{35}$ and Tolerance, Annoyance, Cut down attempts, Eye opener (T-ACE) and its revision, the T-ACER3. ${ }^{36}$ Several of these screening tools, though, were not developed specifically for use in pregnant women (CAGE, MAST) and were designed to pick up alcohol use patterns more common among men, especially alcohol dependence. Therefore, they are less effective in identifying problem drinking in women, let alone pregnant women in whom a strict diagnosis of alcohol dependence is less common. ${ }^{37}$ Moreover, positive cut-off scores for alcohol use disorders screening tools need to be set differently for women than for men, since relative to men, women experience higher blood alcohol levels at identical exposures and women are more susceptible to end organ damage resulting from alcohol (for example, cardiomyopathy). ${ }^{40,38,39}$

However, the T-ACE/T-ACER-3, TWEAK, and Substance Use Risk Profile-Pregnancy (SURP-P) have been empirically validated specifically for use in pregnant women, with the T-ACE/T-ACER-3 bearing the recommendation of both the American College of Obstetrics and Gynecology and the National Institute on Alcohol Abuse and Alcoholism for AUD screening in pregnant women. ${ }^{40,41,42}$ It is important to note that both the T-ACE/T-ACER-3 and the TWEAK were validated to assess for at-risk drinking as defined as $>1$ ounce of alcohol consumed per day.

The T-ACE was developed by an obstetrician specifically for use in obstetric-gynecologic practices, and its three yes/no questions (Have people Annoyed you by criticizing your drinking? Have you felt that you ought to Cut down on your drinking? Have you had to have a drink first thing in the morning to steady your nerves or get rid of a hangover-Eye Opener?) and one quantifying question (Tolerance: How many drinks does it take to make you feel high?) take less than 1 minute to administer, making it practical and efficient. A positive screen is an answer of "yes" to two or more of the A, C, or E screening questions (2 points are assessed if the woman indicates that it takes 2 or more drinks for her to feel high in response to the $\mathbf{T}$ question), with studies indicating it to be $69 \%$ sensitive and $89 \%$ specific among a cohort of 971 study participants. In the same 971 study participants, the TACE was found to be superior relative to the $38 \%$ and $92 \%$ sensitivity and specificity, respectively, of the CAGE, and the $36 \%$ and $96 \%$ sensitivity and specificity, respectively, of the MAST. ${ }^{43}$ 
The "T-ACER-3" is the same screening questionnaire as the T-ACE, but the positive test cut-off has been increased from the traditional 2 points to 3 points. Increasing the cut-off to 3 points resulted in greater specificity (decreased false positives, which can be time consuming for the clinician to further investigate) while maintaining sensitivity, and was also more effective in identifying women whose children had FASD. ${ }^{44}$

The 5-question TWEAK screening tool draws heavily from the MAST, CAGE, and T-ACE tools that came before it: Tolerance (How many drinks can you hold?), Worry (Have close friends or relatives Worried or complained about your drinking?), Eye Opener (Do you sometimes take a drink in the morning to wake up?), Amnesia (Has a friend or family member ever told you about things you did or said while you were drinking that you could not remember?), $\mathbf{K}(\mathbf{C})$ (Do you sometimes feel the need to Cut down on your drinking?). ${ }^{45}$ While not specifically designed with pregnant women in mind, the TWEAK has nonetheless been shown to be effective in detecting at risk drinking in pregnant women. ${ }^{46}$ In one recent head-to-head study of TWEAK versus T-ACE in pregnant women, TWEAK was shown to have slightly better specificity than T-ACE (36-43\% versus $19-34 \%$, respectively) in pregnant women calling in to a help line. ${ }^{47}$

Partly in response to the moderate specificity of both the T-ACE and TWEAK, researchers developed the SURP-P as a screening tool for drugs and alcohol use during in pregnant women. ${ }^{48}$ The SURP-P is a 3-question tool with concurrent stratification of pre-test risk that is easily administered and shows high specificity and sensitivity. The three questions of the SURP-P are: 1) Have you ever smoked marijuana, 2) In the month before you knew you were pregnant, how many beers, how much wine, or how much liquor did you drink ( $0=$ low risk, 1=moderate risk, 2-3=high risk), and 3) Have you ever felt that you needed to cut down on your drug or alcohol use? In low-risk populations, one or more affirmative items constitutes a positive screen, with good sensitivity (91\% versus $63 \%$ for the TWEAK in this study). In high risk populations, two or more affirmative items marks a positive screen, with the SURP-P demonstrating high sensitivity in this population although not much more sensitive than the TWEAK ( 88 versus $86 \%$, respectively). The SURP-P, therefore, has the advantage of allowing results to be measured against a backdrop of risk stratification that may lead to, overall, fewer false positive screening results.

There are no head-to-head data to suggest that any one screening instrument is superior than the others in identifying AUDs in pregnant women. What is clinically important is that all women of child-bearing-age, especially those who are known to be pregnant are systematically screened for the presence of AUDs. The T-ACE/T-ACER-3, TWEAK, and SURP-P have all been validated for use in screening pregnant women for the presence of AUDs.

\section{Pharmacologic Interventions for AUDs During Pregnancy}

Currently, there are three medications approved for the treatment of AUDs by the Food and Drug Administration (FDA) in the United States: naltrexone, disulfiram, and acamprosate. Naltrexone is a mu opioid receptor antagonist that has been shown to decrease the risk of heavy drinking to $83 \%$ of the risk in placebo groups and decrease drinking days by about 
$4 \% .{ }^{49}$ Naltrexone is available in both oral and long-acting injectable formulations. However, there are no published studies on the safety or efficacy of either formulation of naltrexone for use in AUD in pregnant women. Naltrexone is classified as a category $\mathrm{C}$ medication by the FDA, meaning that animal studies have shown adverse effects on the fetus, but there are no adequate studies on reproductive effects and safety in human pregnancy. ${ }^{50}$ Naltrexone's other indication is in the treatment of opioid use disorders (OUDs), and data from studies of pregnant women with OUD treated with naltrexone have not shown ill-effects on birth outcomes, but the long-term effects on pre-and post-natal development are not wellknown. ${ }^{51}$ In particular, the endogenous opioid system is active during fetal development, and the long-term effects of antagonizing this system during development are not well known. ${ }^{53}$

Disulfiram (an aldehyde dehydrogenase inhibitor that results in a severe reaction when alcohol is consumed concurrently with it, resulting in a strong deterrent effect) is also a category $\mathrm{C}$ medication. There is some evidence, albeit inconsistent, that exposure to disulfiram in the first trimester may increase the risk of fetal malformations. ${ }^{52,53}$

Furthermore, the intensity of the disulfiram-alcohol reaction, which can involve severe acute autonomic instability, including hypertension, can also be considered a risk to the pregnant woman and her fetus, although there have been no studies specifically assessing the magnitude of this particular risk.

Acamprosate, which is believed to exert its action through modulation of glutamate neurotransmission thereby reducing post-acute withdrawal symptoms and consequently helping to maintain sobriety, ${ }^{54}$ is also a category $\mathrm{C}$ medication. Animal data suggest possible teratogenic effects of acamprosate, ${ }^{55}$ but there are no human trial data to support this.

Therefore, when deciding whether to use a medication to assist in the treatment of an AUD in a pregnant woman, the risks posed by the use of alcohol itself must be carefully weighed against the risks of the medications themselves.

\section{Behavioral Interventions for AUDs During Pregnancy}

Validated behavioral interventions for AUDs in pregnancy include many of those employed in the treatment of non-pregnant individuals, such as motivational enhancement therapy, ${ }^{56}$ brief interventions, ${ }^{57,58}$ and cognitive behavioral therapies. ${ }^{59}$ However, the data do not suggest that any one particular mode of behavioral intervention is superior to the others in pregnant women with AUDs. For instance, a recent Cochrane systematic review pooled randomized control trial data of educational and psychological interventions for reducing drinking during pregnancy. The psychological interventions included CBT, brief psychodynamic psychotherapy, interpersonal psychotherapy, and supportive counseling/ therapy, and the educational interventions included brief educational counseling sessions, structures long-term educational programs with motivational enhancement, individualfocused educational strategies, family-focused programs, professional group education and self-help group educational interventions. The authors concluded that there were not enough data to determine overall effectiveness of psychological and/or educational interventions for 
reducing alcohol consumption in pregnant women. While it is difficult to determine effectiveness from pooled data in a systematic review, the authors also indicate that the individual studies themselves suggest that either psychological or educational interventions "may encourage women to abstain from alcohol in pregnancy." ${ }^{60}$ Suffice it to say, therefore, that psychosocial interventions canform an important part of treatment for any pregnant patient suffering from an AUD, in the same way that they would for a non-pregnant patient with AUD. ${ }^{61}$

It is often in the context of engaging in psychosocial treatments that women may discover that they are pregnant. Furthermore, the revelation of being pregnant may not only cause turmoil for the patient, but for many caregivers the perceived higher risks and stakes associated with treating a pregnant woman can be anxiety-provoking.

As the epidemiological data above suggest, it is relatively common for women to consume alcohol during pregnancy, especially early in the pregnancy; however, it is relatively uncommon for pregnant women to present with either severe AUD or heavy alcohol use. It is these particular women, though, when they do present in treatment, who can cause a great deal of concern in their providers. Clinical experience tells us that pregnant women with substance use disorders commonly present with a host of other serious psychosocial issues such as homelessness, co-occurring mental health disorders, trauma, domestic violence, and other substance use disorders, to name a few. Therefore, social work and case management services can become an integral part of the treatment of pregnant women with substance use disorders, perhaps even more so than with non-pregnant women.

\section{Alcohol Withdrawal in Pregnancy}

Alcohol withdrawal occurs with the sudden cessation of alcohol consumption in someone who has sustained alcohol intake, and is marked by a constellation of physiologic instabilities that range from minor restlessness and tremor to more severe symptoms such as hypertension, tachycardia, seizures, hyperthermia, hallucinations and even death. ${ }^{62}$

Withdrawal phenomena are believed to result, in part, from neuronal hyperactivity mediated through the excitatory neurotransmitter glutamate. Chronic exposure to the inhibitory effects of alcohol, which acts principally through neuronal gamma-aminobutyric acid (GABA) receptors, stimulates a compensatory up-regulation of N-methyl-D-aspartate (NMDA) receptors and, consequently, glutamate transmission. ${ }^{63}$ Once unopposed by the chronic inhibitory effects of alcohol, the up-regulated excitatory system functions hyperactively, resulting in neurotoxicity that accounts for the spectrum of clinical withdrawal symptoms. Therefore, alcohol withdrawal is oftentimes considered a medical emergency, especially in pregnancy.

There are few data on the effects of acute alcohol withdrawal in pregnancy, and for understandable reasons studying this phenomenon in a controlled manner is ethically untenable. However, by extrapolation, we can make certain assumptions about the risks associated with alcohol withdrawal on mother and fetus. Studies have demonstrated that physiologic and psychological stress during pregnancy can have deleterious effects on mother and fetus, such as preterm birth and low birth weight. ${ }^{64,65}$ In non-pregnant 
individuals, the hypothalamic-pituitary-adrenal (HPA) axis is believed to modulate one's response to environmental stress and is mediated through the release of corticotrophin releasing hormone (CRH) by the hypothalamus. CRH then stimulates the release of adrenocorticotropin hormone (ACTH) by the pituitary gland, which in turn stimulates the release of cortisol by the adrenal glands. Cortisol then mediates downstream physiologic stress responses such as increasing blood glucose levels via gluconeogenesis, regulating the body's $\mathrm{pH}$ balance via sodium and potassium metabolism, weakening immune responses, and also negatively feeding back onto the pituitary gland and hypothalamus, effectively shutting down the stress HPA axis. ${ }^{66}$

In pregnancy, though, cortisol can actually increase the production of CRH in the placenta, providing a positive systemic feedback loop. Premature increases in systemic CRH levels, before 20 weeks gestation in particular, have been tied to a cascade of fetal maturational signals (via CRH and estrogens) that may lead to preterm labor. ${ }^{67,68}$ Chronic heavy drinking is known to alter the dynamics of the HPA axis, and alcohol withdrawal, in particular, has been shown to induce a hypercortisol state that may also mediate many of the clinical symptoms of alcohol withdrawal. ${ }^{69,70,71}$ Therefore, pregnant women in alcohol withdrawal may be uniquely vulnerable to the effects of alcohol withdrawal, although this relationship is unstudied formally.

Moreover, regardless of etiology, hypertension is considered a significant risk during pregnancy. Specifically, placental perfusion is maintained through a balance of maternal and fetal factors. Maternal hypertension, especially after 20 weeks, is known to alter the dynamics of the placental circulatory system. ${ }^{72}$ Again, there are no studies specifically addressing the effects of alcohol withdrawal-related hypertension, but it stands to reason that hypertension alone, as a consequence of withdrawal, may present its own acute risks to both the mother and the fetus.

Given these risks outlined above, the management of pregnant women with physiological dependence on alcohol likely warrants an even more careful and perhaps intensive, monitored approach to management than other patients.

\section{Treatment of alcohol withdrawal in pregnancy}

Since acute alcohol withdrawal results from the abrupt cessation of the tonic GABAergic effects of alcohol, treatment for alcohol withdrawal has traditionally centered on careful replacement with alternative GABAergic substances and controlled down-titration. Therefore, medications that modulate the GABA receptor system have been employed in detoxification efforts, such as barbiturates and benzodiazepines, with the latter in particular having emerged as the most common medication of choice in the United States for the past several decades.

There is a dearth of evidence to guide decisions regarding management of acute alcohol withdrawal in pregnancy. Since the standard medical treatment for alcohol withdrawal is benzodiazepines, examining the evidence for safety of benzodiazepines, in general, in pregnancy may be instructive. 
Early case studies regarding the use of benzodiazepines in pregnancy suggested that these medications may be associated with increased risk of fetal major malformations (MM); this has consequently shaped treatment recommendations ever since. ${ }^{73}$ A 1998 meta-analysis by Dolovich et al. which included 11 cohort studies and 12 case control studies examining the relationship between use of benzodiazepines and MM revealed mixed results. The cohort studies showed no difference between exposure to benzodiazepines and occurrence of MM, while the case control studies demonstrated an increased risk for MM and cleft palate (in particular) with perinatal benzodiazepine exposure: odds ratio of 3.01 for all MM and 1.79 for cleft palate. ${ }^{74}$ This meta-analysis was updated in 2011 to include 3 additional cohort studies, and there remained no difference in rates of MM between exposed and non-exposed groups among these cohort studies. ${ }^{75}$

These findings should be viewed with caution, since the studies included in the metaanalyses did not distinguish between individual benzodiazepines used, dosages given, gestational age at exposure, or concurrent substances used. Additionally, the fact that the case-control studies showed effect and the cohort studies did not calls into question the possibility of recall bias. Cohort studies select a population of people with similar exposures and track them in time to determine who, of the exposed, develop a condition of interest. Cohort studies allow for the calculation of absolute risk for developing a condition (like MMs) relative to exposure to some possible agent (like benzodiazepines). On the other hand, case-control studies start by identifying affected individuals (cases) and compare their potential exposures with those of similarly matched unaffected individuals (controls). Since case control studies rely so much on individual recall, they are subject to recall bias (systematic error in recollection of information by study participants); therefore, it is difficult to draw conclusions about causal relationships based only on the results of case control studies.

Furthermore, the difference in effect noted between the case-control studies and cohort studies above could be further influenced by the non-random misclassification bias known as surveillance bias, or "unmasking bias."Surveillance bias, simple put, is governed by the principle that "the more you look, the more you find." 76 The danger with surveillance bias is that one group of individuals in a study may be followed more closely than another; as a result, the more closely monitored group may have an outcome diagnosed more often than the less closely followed, group. Case-control study designs can be vulnerable to this form of non-random bias. In the situation above, therefore, it is conceivable that the risk of MM in the case-control studies may have been over-estimated due to surveillance bias; women who were identified as affected may have then been subject to greater scrutiny of their exposures, resulting in an inflated attribution of risk relative to the non-affected arm.

Bellantuono et al. systematically reviewed the relationship between individual types of benzodiazepines and fetal MM. ${ }^{77}$ The review included a total of 12 studies, each of which suffered from the same limitations highlighted in the Dolovich meta-analysis above: lack of information regarding duration of exposure, dosing levels, and concurrent substance use. Nonetheless, the data pointed to no conclusive link between alprazolam, clonazepam, chlordiazepoxide, or diazepam exposure in the first trimester and development of MM. A closer look at these data, though, is warranted. The authors included one study of alprazolam 
in which the mean exposure dose was $29.8 \mathrm{mg}$ (overdoses in suicide attempts; $\mathrm{n}=30$ ). In this alprazolam overdose-exposed population, a $23.3 \%$ first trimester spontaneous abortion rate was noted; however, no higher risk of MM was observed in surviving infants. Given the nature of the exposure (acute overdose), it is difficult to extrapolate risk of alprazolam exposure at typical therapeutic dose ranges from these data.

Two studies of first trimester clonazepam exposure during pregnancy revealed no increased risks of MM. The first study ( $\mathrm{n}=73$ ) examined exposures at a dose of $2 \mathrm{mg}$ daily, and a second study ( $\mathrm{n}=71)$ did not specify exposure dose. From this data it is again difficult to draw definitive conclusions about the risk of first trimester clonazepam exposure and development of MM. In distinction to the alprazolam data, though, at least some of this data derives from exposures at typical prescription dosing.

One case-control study $(\mathrm{n}=201)$ showed a preliminary increase in risk of cardiovascular malformations associated with first trimester chlordiazepoxide exposure, but the authors posit a significant recall bias contributing to this finding. Similar to the data above regarding alprazolam, one study looking at the risk of MM in women with first trimester chlordiazepoxide overdoses showed no relationship; however, a high rate of spontaneous abortion was cited in these women (13.6\%).

Four studies looking at the relationship between diazepam and MM have not revealed significantly elevated risks of MM. One study $(n=31)$ showed no increased risk, specifically, with exposure to diazepam 10mg twice a day. Again, large doses of diazepam in suicide attempts $(25-800 \mathrm{mg})$ were associated with high rates of spontaneous abortion (40/229 cases).

One case-control study of lorazepam $(\mathrm{n}=262)$ use in the first trimester was linked to a slight increase in anal atresia (20/10,000 versus a 3/10,000 congenital rate).

In aggregate, from these data systematically reviewed by Bellantuono it is difficult to formulate causal relationships or recommendations regarding use of one benzodiazepine relative to another during pregnancy.

Regardless of biases that may account for the observed differences between the case- control and cohort studies in the Dolovich meta-analysis or the Bellantuono systematic review, the fundamental factor that makes determination of any relationship between benzodiazepine exposure and MM most difficult is the lack of controlling for confounding factors in the included studies themselves, particularly concurrent exposure to other substances in addition to benzodiazepines. It is conceivable, therefore, that the modest increased risks for anal atresia or MM (case-control studies) could be attributable to non-benzodiazepine substance exposures.

A recent Swedish National Health Registry review explored the relationship between benzodiazepines and congenital malformations. ${ }^{78}$ Data from 1000 infants born to mothers using benzodiazepines alone during pregnancy showed no higher risk for severe congenital malformations or cardiac defects when compared to the general public. Furthermore, one study that followed up with 550 children who had been exposed to benzodiazepines in utero 
up to 4 years of age, and showed no increase in adverse effects on neurobehavioral development or intelligence quotient. ${ }^{79}$

Additional considerations are the so-called "floppy infant syndrome" and neonatal benzodiazepine withdrawal. Floppy infant syndrome is characterized by a constellation of symptoms that may include mild sedation, hypotonia, reluctance to suck, apneic spells, and/or cyanosis that can persist for hours to months after birth in infants born to mothers using sedative-hypnotic medications at the time of delivery. Neonatal benzodiazepine withdrawal can consist of "hypertonia, hyperreflexia, restlessness, irritability, abnormal sleep patterns, inconsolable crying, tremors or jerking of the extremities, bradycardia, cyanosis, suckling difficulties, apnea, risk of aspiration of feeds, diarrhea and vomiting, and growth retardation." 80 The etiologies of floppy infant syndrome and neonatal benzodiazepine withdrawal are proposed to be due to the physiologic effects of intoxication or withdrawal from benzodiazepines in the newborn, respectively. There are no studies suggesting that using a long acting (i.e., clonazepam) versus a short acting (i.e., lorazepam) benzodiazepine reduces the risk of developing either floppy infant syndrome or neonatal benzodiazepine withdrawal.

In sum, the data regarding fetal risks associated with in utero exposure to benzodiazepines is mixed and inconclusive. Early data suggesting a link between benzodiazepine use in pregnancy and $\mathrm{MM}$ are difficult to draw conclusions from and these findings have not been conclusively reaffirmed by subsequent studies. If a benzodiazepine is clinically warranted during pregnancy, there are not enough data to guide decisions about which particular benzodiazepine to use, how to dose it, or how to advise women definitively about the risks between the different benzodiazepines. If the decision is made to discontinue benzodiazepines that a pregnant woman is already taking, it is critical that this be done in a careful and judicious manner to avoid benzodiazepine withdrawal, since benzodiazepines can also induce physiologic dependence and can carry risks similar to those incurred with alcohol withdrawal.

Furthermore, there are no studies that have specifically examined the effects of acute, shortterm, administration of benzodiazepines as is commonly used in the treatment of alcohol withdrawal (detoxification). Therefore, in the absence of clear evidence, the clinician must carefully weigh the theoretical risks and potential benefits of benzodiazepine use/exposure during pregnancy against the potential risks posed by alcohol withdrawal as highlighted above.

\section{Conclusion}

It is beyond the scope of this review to provide specific treatment recommendations or algorithms. This paper is not a comprehensive review of all published studies with systematic data comparisons, which can be considered a limitation of the review. Rather, the aim is to provide an overview of the complexity of the treatment considerations in this clinically challenging cohort. 
The presence of an AUD during pregnancy presents many clinical challenges, and there is little evidence to guide management decisions. Encouragingly, pregnancy seems to be a time of relatively high motivation for change and success in accomplishing it, as evidenced by the lower prevalence of AUDs in pregnant women relative to their non-pregnant female peers. A spectrum of dysmorphologies and downstream cognitive and behavioral deficits has been linked to maternal alcohol consumption, but there are conflicting data on at what level of consumption the risks of these deleterious effects of alcohol increase. Maternal decisions to drink appear to be shaped by social norms, which can be informed by public policy statements. Policies regarding alcohol use during pregnancy vary from country to country, and efforts to shape public health guidelines about drinking in pregnancy may have significant effects on prevalence. While there are few data on safety or efficacy on pharmacological interventions for AUDs in pregnancy, whatever potential risks posed by disulfiram, naltrexone, or acamprosate should be weighed against the risks of ongoing alcohol use. Behavioral interventions should form the cornerstone of treatment of AUDs in pregnancy. However, there are no data indicating that one behavioral approach is better, per se, than another in expectant mothers. Therefore, decisions regarding behavioral and psychopharmacologic interventions should be made on a case-by-case basis depending on the patient's unique needs and best therapeutic fit to meet those needs. Acute alcohol withdrawal poses a threat to both mother and fetus, and should be treated as a medical emergency, as it is for any person. However, pregnant women may be uniquely vulnerable to the deleterious effects of alcohol withdrawal and may require more intensive monitoring and ongoing evaluation by obstetrical experts. The data on the safety of benzodiazepines in pregnancy are scant and conflicting, and there are no data specifically addressing the safety of benzodiazepines in short-term, high-dose tapers as are often employed in alcohol detoxification. The pregnant woman with an AUD, and particularly one requiring detoxification, can present with myriad social and psychological co-morbidities that may call for a team approach incorporating psychiatric, social work, obstetrical, and addictions expertise.

\section{Acknowledgements}

Dr. Weiss is supported by grants K24 DA022288 and U10 DA15831 from the National Institute on Drug Abuse.

\section{References}

1. Substance Abuse and Mental Health Services Administration. Rockville, MD: Substance Abuse and Mental Health Services Administration; 2013. Results from the 2012 National Survey on Drug Use and Health: Summary of National Findings. NSDUH Series H-46, HHS Publication No. (SMA) 13-4795.

2. Substance Abuse and Mental Health Services Administration, Office of Applied Studies. Rockville, MD: 2008 Sep 11. The NSDUH Report - Alcohol Use among Pregnant Women and Recent Mothers: 2002 to 2007.

3. Substance Abuse and Mental Health Services Administration. Rockville, MD: Substance Abuse and Mental Health Services Administration; 2013. Results from the 2012 National Survey on Drug Use and Health: Summary of National Findings. NSDUH Series H-46, HHS Publication No. (SMA) 13-4795.

4. [Accessed 10 March 2014] Results from the 2011 National Survey on Drug Use and Health: Summary of National Findings. Available on http://www.samhsa.gov/data/NSDUH/2k11Results/ NSDUHresults2011.htm\#3.1.3. 
5. Alvik A, Heyerdahl S, Haldorsen T, Lindemann R. Alcohol use before and during pregnancy: a population-based study. Acta Obstetricia et Gynecologica. 2006; 85:1292-1298.

6. Substance Abuse and Mental Health Services Administration. [Accessed 18 March 2013] The NSDUH report: data spotlight. 18 percent of pregnant women drink alcohol during early pregnancy. 2013 Sep 9. Available on: http://www.samhsa.gov/data/spotlight/spot123-pregnancyalcohol-2013.pdf.

7. Lemoine P. The history of alcoholic fetopathies. J Popul Ther Clin Pharmacol. 2012; 19(2):224226.

8. Lemoine P, Harousseau H, Borteyru JP, Menuet JC. Children of alcoholic parents-observed anomalies: discussion of 127 cases. Ouest Med. 1968; 8:476-482.

9. Jones KL, Smith DW, Ulleland CN, Streissguth AP. Pattern of malformation in offspring of chronic alcohol mothers. The Lancet. 1973; 301(7815):1267-1271.

10. Patra J, Bakker R, Irving H, Jaddoe VWV, Malini S, Rehm J. Dose-response relationship between alcohol consumption before and during pregnancy and the risks of low birth weight, preterm birth and small-size-for-gestational age (SGA) —A systematic review and meta-analysis. BJOG. 2011; 118(12):1411-1421. [PubMed: 21729235]

11. Blume SB. Is social drinking during pregnancy harmless? There is reason to think not. Adv Alcohol Subst Abuse. 1985; 5(1-2):209. [PubMed: 3832898]

12. Sood B, Delaney-Black V, Covington C, Nordstrom-Klee B, Ager J, Templin T, Janisse J, Martier S, Sokol RJ. Prenatal alcohol exposure and childhood behavior at age 6 to 7 years: I. doseresponse effect. Pediatrics. 2001; 108(2):e34. [PubMed: 11483844]

13. Streissguth AP, Sampson PD, Barr HM. Neurobehavioral dose-response effects of prenatal alcohol exposure in humans from infancy to adulthood. Ann NY Acad Sci. 1989; 562:145-158. [PubMed: 2742272]

14. O’Callaghan FV, O’Callaghan M, Najman JM, Williams GM, Bor W. Prenatal alcohol exposure and attention, learning and intellectual ability at 14 years: A prospective longitudinal study. Early Hum Dev. 2007; 83(2):115-123. [PubMed: 16842939]

15. Alata R, Macleod J, hickman M, Sayal K, May M, Smith GD, Lawlor DA. Intrauterine exposure to alcohol and tobacco use and childhood IQ: findings from a parental-offspring comparison within the Avon Longitudinal Study of parents and children. Ped Res. 2008; 64(6):659-656.

16. Kelly YJ, Sacker A, Gray R, Kelly J, Wolke D, Head J, Quigley MA. Light drinking during pregnancy: still no increased risk for socioeconomic difficulties or cognitive deficits at 5 years of age? J Epidemiol Community Health. 2012; 66:41e48. [PubMed: 20924051]

17. Testa M, Quigley BM, Eiden RD. The effects of prenatal alcohol exposure on infant mental development: a meta-analytical review. Alcohol Alcohol. 2003; 38:295-304. [PubMed: 12814894]

18. Sayal K, Heron J, Golding J, Emond A. Prenatal alcohol exposure and gender differences in childhood mental health problems: a longitudinal population-base study. Pediatrics. 2007; 119(2):e426-e434. [PubMed: 17272604]

19. Coles CD, Smith IE, Falek A. Prenatal alcohol exposure and infant behavior: Immediate effects and implications for later development. Advances in Alcohol and Substance Abuse. 1987; 6:87104. [PubMed: 3425480]

20. Makarechian N, Agro K, Devlin J, Trepanier E, Koren G, Einarson TR. Association between moderate alcohol consumption during pregnancy and spontaneous abortion, stillbirth and premature birth: A meta-analysis. Canadian Journal of Clinical Pharmacology. 1998; 5:169-176.

21. Polygenis D, Wharton S, Malmberg C, Sherman N, Kennedy D, Koren G, Einarson TR. Moderate alcohol consumption during pregnancy and the incidence of fetal malformations: A meta-analysis. Neurotoxicology and Teratology. 1998; 20:61-67. [PubMed: 9511170]

22. Morrow-Tlucak M, Ernhart CB, Sokol RJ, Martier S, Ager J. Underreporting of alcohol use in pregnancy: Relationship to alcohol. Alcoholism: Clinical and Experimental Research. 1989; 13:399-401.

23. [Accessed 1 March 2014] United States Public Law 100-690: Alcohol and Drug Traffic Safety Act of 1988. Available on: http://www.gpo.gov/fdsys/granule/STATUTE-102/STATUTE-102Pg4181/content-detail.html 
24. National Institute for Health and Clinical Excellence. Antenatal Care: NICE clinical guidelines 62. 2010 Jun. Available on: http://www.nice.org.uk/nicemedia/live/11947/40115/40115.pdf.

25. From: International Guidelines on Drinking and Pregnancy. Available on: http://www.icap.org/ Table/InternationalGuidelinesOnDrinkingAndPregnancy.

26. Dell, CA.; Roberts, G. Research update: alcohol use in pregnancy: an important Canadian public health and social issue. Ottawa: Public Health Agency of Canada; 2005.

27. Callinan, S.; Room, R. Alcohol consumption during pregnancy: results from the 2010 National Drug Strategy Household Survey. Canberra: Centre for Alcohol Policy Research; 2012.

28. Nykjaer C, Alwan NA, Greenwood DC, Simpson NAB, Hay AWM, White KLM, Cade JE. Maternal alcohol intake prior to and during pregnancy and risk of adverse birth outcomes: evidence from a British cohort. Epidemiol Community Health. 2014; 68:542-549.

29. Centers For Disease Control and Prevention. [accessed July 2014] Fetal alcohol spectrum disorders (FASDs)—United States 2014. Available at: http://www.cdc.gov/NCBDDD/fasd/data.html

30. Newnham C. No alcohol in pregnancy is the safest choice for women who are, or might become pregnant. Premie Press: a quarterly publication for those interested in the development of premature babies and children. 2012; 13:8-9. Available at: http://search.informit.com.au/ documentSummary; dn=894614457788047;res=IELHEA.

31. Jones SC, Teleta J. What influences Australian women to not drink alcohol during pregnancy? Australian Journal of Primary Health. 2012; 18:68-73. [PubMed: 22394665]

32. Ewing JA. Detecting Alcoholism: The CAGE Questionnaire. JAMA. 1984; 252:1905-1907. [PubMed: 6471323]

33. Saunders JB, Aasland OG, Babor TF, et al. Development of the Alcohol Use Disorders Identification Test (AUDIT): WHO Collaborative Project on Early Detection of Persons with Harmful Alcohol Consumption-II. Addiction. 1993; 88:791-804. [PubMed: 8329970]

34. Selzer ML. The Michigan Alcoholism Screening Test: The quest for a new diagnostic instrument. American Journal of Psychiatry. 1971; 127(12):89-94.

35. Russel M, Martier SS, Sokol RJ, Mudar P, Bottoms S, Jacobson S, Jacobson J. Screening for Pregnancy Risk-Drinking. Alcoholism: Clinical and Experimental Research. 1994; 18(5):11561161.

36. Chang G, Wilkins-Haug L, Berman S, et al. Alcohol use and pregnancy: improving identification. Obstet Gynecol. 1998; 91:892-898. [PubMed: 9610992]

37. Chang, G. [accessed 5 July 2014] Alcohol-screening instruments for pregnant women. National Institute on Alcohol Abuse and Alcoholism Publications. Available on: http://pubs.niaaa.nih.gov/ publications/arh25-3/204-209.htm

38. Graham K, Wilsnack R, Dawson D, Vogeltanz N. Should alcohol consumption measures be adjusted for gender differences? Addiction. 1998; 93:1137-1147. [PubMed: 9813895]

39. Urbano-Marquez A, Estruch R, Fernandez-Sola J. The greater risk of alcoholic cardiomyopathy and myopathy in women compared with men. Journal of the American Medical Association. 1995; 274:149-154. [PubMed: 7596003]

40. Jones TB, Bailey BA, Sokol RJ. Alcohol use in pregnancy: insights in screening and intervention for the clinician. Clin Obstet Gynecol. 2013; 56(1):114-123. [PubMed: 23314712]

41. Dawson DA, Grant BF, Stinson FS, Zhou Y. Effectiveness of the derived alcohol use disorders test (AUDIT-C) in screening for alcohol use disorders and risk drinking in the US general population. Alcohol Clin Exp Res. 2005; 29:844-854. [PubMed: 15897730]

42. Chang G, Wilkins-Haug L, Berman S, et al. Alcohol use and pregnancy: improving identification. Obstet Gynecol. 1998; 91:892-898. [PubMed: 9610992]

43. Sokol RJ, Martier SS, Ager JW. The T-ACE questions: Practical prenatal detection of riskdrinking. American Journal of Obstetrics and Gynecology. 1989; 160:863-871. [PubMed: 2712118]

44. Chiodo LM, Delaney-Black V, Sokol RJ, Janisse J, Pardo Y, Hannigan JH. Increased cut-point of the TACER-3 screen reduces false positives without losing sensitivity in predicting risk alcohol drinking in pregnancy. Alcohol Clin Exp Res. 2014; 38(5):1401-1408. [PubMed: 24655071] 
45. Chan AK, Pristach EA, Welte JW, Russell M. The TWEAK test in screening for alcoholism/heavy drinking in three populations. Alcoholism: Clinical and Experimental Research. 1993; 6:11881192.

46. Russell M, Martier SS, Sokol RJ. Detecting risk drinking during pregnancy: A comparison of four screening questionnaires. American Journal of Public Health. 1996; 86:1435-1439. [PubMed: 8876514]

47. Sarkar M, Einarson T, Koren G. Comparing the effectiveness of TWEAK and T-ACE in determining problem drinkers in pregnancy. Alcohol Alcohol. 2010 Jul-Aug;45(4):356-360. [PubMed: 20497951]

48. Yonkers KA, Gotman N, Kershaw T, FOrray A, Howell HB, Rounsaville BJ. Screening for prenatal substance use: development of the Substance Use Risk Profile-Pregnancy scale. Obstetrics and Gynecology. 2010; 116(4):827-833. [PubMed: 20859145]

49. Rösner S, Hackl-Herrwerth A, Leucht S, Vecchi S, Srisurapanont M, Soyka M. Opioid antagonists for alcohol dependence. Cochrane Database Syst Rev. 2010 Dec.8(12):CD001867. [PubMed: 21154349]

50. Zagon IS, Tobias SW, Hytrek SD, McLaughlin PJ. Opioid receptor blockade throughout prenatal life confers long-term insensitivity to morphine and alters mu opioid receptors. Pharmacol Biochem Behav. 1998; 59:201-207. [PubMed: 9443556]

51. Jones HE, Chisolm MS, Jansson LM, Terplan M. Naltrexone in the treatment of opioid-dependent pregnant women: the case for a considered and measured approach to research. Addiction. 2013; 108(2):233-247. [PubMed: 22471668]

52. Nora H, Nora J, Blu J. Limb reduction anomalies in infants born to disulfiram-treated alcohol mothers. Lancet. 1977; 3:664-669. [PubMed: 71482]

53. Helmbrecht GD, Hoskins IA. First trimester disulfiram exposure: report of two cases. Am J Perinatol. 1993; 10(1):5-7. [PubMed: 8442800]

54. Rösner S, Hackl-Herrwerth A, Leucht S, Lehert P, Vecchi S, Soyka M. Acamprosate for alcohol dependence. Cochrane Database Syst Rev. 2010 Sep 8.(9):CD004332. [PubMed: 20824837]

55. Product monograph, Campral Acamprosate calcium, delayed release tablets $333 \mathrm{mg}$, alcohol abstinence aid; Mylan pharmaceuticals ULC. 2011 Sep 8.

56. Ingersoll KS, Ceperich SD, Hettema JE, Farrell-Carnahan L, Penberthy JK. Preconceptional motivational interviewing interventions to reduce alcohol-exposed pregnancy risk. J Subst Abuse Treat. 2013; 44(4):407-416. [PubMed: 23192220]

57. O'Connor MJ, Whaley SE. Brief intervention for alcohol use by pregnant women. Am J Public Health. 2007; 97:252-258. [PubMed: 17194863]

58. Chang G, Goetz MA, Wilkins-Haug L, Berman S. Brief intervention for prenatal alcohol use: an in depth look. J Subst Abuse Treat. 2000; 18:365-369. [PubMed: 10812310]

59. Yonkers KA, Forray A, Howell HB, Gotman N, Kershaw T, Rounsaville BJ, Carroll KM. Motivational enhancement therapy coupled with cognitive behavioral therapy versus brief advice: a randomized trial for treatment of hazardous substance use in pregnancy and after delivery. Gen Hosp Psychiatry. 2012; 34(5):439-449. [PubMed: 22795046]

60. Stade BC, Bailey C, Dzendoletas D, Sgro M, Dowswell T, Bennett D. Psychological and/or educational interventions for reducing alcohol consumption in pregnant women and women planning pregnancy. Cochrane Database Syst Rev. 2009 Apr 15.(2):CD004228. [PubMed: 19370597]

61. Winhusen T, Kropp F, Babcock D, Hague D, Erickson SJ, Renz C, Rau L, Lewis D, Leimberger J, Somoza E. Motivational enhancement therapy to improve treatment utilization and outcome in pregnant substance users. J Subst Abuse Treat. 2008; 35(2):161-173. [PubMed: 18083322]

62. Hall W, Zador D. The alcohol withdrawal syndrome. The Lancet. 1997; 349:1897-1900.

63. Sanna E, Mostallino MC, Busonero F, Talani G, Tranquilli S, Mameli M, Spiga S, Follesa P, Biggio G. Changes in GABA(A) receptor gene expression associated with selective alterations in receptor function and pharmacology after ethanol withdrawal. J Neurosci. 2003; 23:11711-11724. [PubMed: 14684873]

64. Enlow MB, Kullowatz A, Staudenmayer J, Spasojevic J, Ritz T, Wright RJ. Associations of maternal lifetime trauma and perinatal traumatic stress symptoms with infant cardiorespiratory 
reactivity to psychological challenge. Psychosom Med. 2009; 71(6):607-614. [PubMed: 19553287]

65. Hobel CJ, Goldstein A, Barrett ES. Psychosocial stress and pregnancy outcome. Clin Obstet Gynecol. 2008; 51(2):333-348. [PubMed: 18463464]

66. Stephens MA, Wand G. Stress and the HPA Axis: Role of Glucocorticoids in Alcohol Dependence. Alcohol Res. 2012; 34(4):468-483. [PubMed: 23584113]

67. McGregor JA, Jackson GM, Lachelin GC, Goodwin TM, Artal R, Hastings C, Dullien V. Salivary estriol as risk assessment for preterm labor: a prospective trial. Am J Obstet Gynecol. 1995; 173:1337-1342. [PubMed: 7485350]

68. Hobel CJ, Arora CP, Korst LM. Cortico-trophin-releasing hormone and CRH-binding protein: differences between patients at risk for preterm birth and hypertension. Ann N Y Acad Sci. 1999; 897:54-65. [PubMed: 10676435]

69. Adinoff B, Iranmanesh A, Veldhuis J, Fisher L. Disturbances of the stress response: the role of the HPA axis during alcohol withdrawal and abstinence. Alcohol Health Res World. 1998; 22:67-72. [PubMed: 15706736]

70. Keedwell PA, Poon L, Papadopoulos AS, Marshall EJ, Checkley SA. Salivary cortisol measurements during a medically assisted alcohol withdrawal. Addict Biol. 2001; 6:247-256. [PubMed: 11900603]

71. Heinz A, Rommelspacher H, Graf KJ, Kurten I, Otto M, Baumgartner A. Hypothalamic-pituitarygonadal axis, prolactin, and cortisol in alcoholics during withdrawal and after three weeks of abstinence: Comparison with healthy control subjects. Psychiatry Res. 1995; 56:81-95. [PubMed: 7792345]

72. Furuya M, Ishida J, Aoki I, Fukamizu A. Pathophysiology of placentation abnormalities in pregnancy-induced hypertension. Vasc Health Risk Manag. 2008; 4(6):1301-1313. [PubMed: 19337544]

73. Saxén I, Saxén L. Associations between maternal intake of diazepam and oral clefts. Lancet. 1975; 13(7933):498. 2. [PubMed: 51304]

74. Dolovich LR, Addis A, Vaillancourt JM, Power JD, Koren G, Einarson TR. Benzodiazepine use in pregnancy and major malformations or oral cleft: meta-analysis of cohort and case-control studies. BMJ. 1998; 317(7162):839-843. [PubMed: 9748174]

75. Enato E, Moretti M, Koren G. The fetal safety of benzodiazepines: an updated meta-analysis. J Obstet Gynaecol Can. 2011; 33(1):46-48. [PubMed: 21272436]

76. Pierce CA, Haut ER, Kardooni S, et al. Surveillance bias and deep vein thrombosis in the National Trauma Data Bank: the more we look, the more we find. J Trauma. 2008; 64(4):932-937. [PubMed: 18404058]

77. Bellantuono C, Tofani S, Di Sciascio G, Santone G. Benzodiazepine exposure in pregnancy and risk of major malformations: a critical review. General Hospital Psychiatry. 2013; 35(1):3-8. [PubMed: 23044244]

78. Reis M, Kallen B. Combined use of selective serotonin reuptake inhibitors and sedatives/hypnotics during pregnancy: risk of relatively severe congenital malformations or cardiac defects. A register study. BMJ Open. 2013; 3(2)

79. McElhatton PR. The effects of benzodiazepine use during pregnancy and lactation. Reprod Toxicol. 1994; 8(6):461-475. [PubMed: 7881198]

80. Iqbal MM, Sobhan T, Ryals T. Effects of Commonly Used Benzodiazepines on the Fetus, the Neonate, and the Nursing Infant. Psychiatric Services. 2002; 53(1):39-49. [PubMed: 11773648] 